\title{
Revisión sistemática: El tratamiento antiagregante previene eventos vasculares oclusivos en pacientes de alto riesgo
}

Review: Antiplatelet therapy prevents occlusive vascular events in high-risk patients ACP Journal Club. 2002 Jul-Aug;137:5.

Antithrombotic Trialists' Collaboration.Collaborative meta-analysis of randomised trials of antiplatelet therapy for prevention of death,myocardial infarction, and stroke in high risk patients. BMJ. 2002 Jan 12; 324:71-86.

\section{Objetivo}

Determinar la efectividad de la terapia antiagregante plaquetaria para la prevención de eventos oclusivos vasculares en pacientes de alto riesgo con enfermedad preexistente

\section{Diseño}

Meta-análisis de ensayos clínicos randomizados controlados disponibles hasta setiembre de 1997

\section{Pacientes}

Los estudios analizados incluían pacientes con eventos vasculares previos o condiciones predisponentes con riesgo aumentado de eventos vasculares oclusivos ( $>3 \%$ al año)

\section{Intervención}

Los pacientes recibían tratamiento antiagregante controlados con placebo o comparados con otro régimen antiagregante

\section{Medición de resultados principales}

El resultado primario era evento vascular mayor: IAM no fatal, ACV no fatal, o muerte por causa vascular o desconocida

\section{Resultados principales}

Se incluyeron 287 estudios de los cuales 197 comparaban terapia antiagregante con placebo, y 90 comparaban diferentes regímenes antiplaquetarios. Para todos los pacientes de alto riesgo, el tratamiento disminuyó la incidencia de eventos vasculares serios en comparación con los controles $(\mathrm{P}<0,001)$ con una reducción relativa del riesgo (RRR) del 19\% [IC 95\%: 16-21].La Reducción también fue significativa para diferentes categorías de riesgo:infarto previo, RRR 21\% (IC 95\%:16-26); ACV o AIT previo, RRR 17\% (IC 95\%:12-21); ACV, RRR 10\% (IC 95\%: 4,3-16);otras condiciones de alto riesgo, RRR 21\% (IC 95\%: 16-26). Se constató un incremento de riesgo para sangrado extracraneal mayor en los pacientes con tratamiento antiagregante plaquetario, OR 1,6 (IC 95\%: 1,4-1,8). Entre los estudios que comparaban diferentes tratamientos antiagregantes, la aspirina resultó superior a regímenes sin AAS. Dosis diarias de 75 a $150 \mathrm{mg}$ fueron tan efectivas como dosis superiores.

\section{Conclusiones}

En pacientes de alto riesgo para eventos oclusivos vasculares mayores, la terapia antiplaquetaria (especialmente la aspirina), reduce la incidencia de IAM no fatal, ACV no fatal, y muerte por causa vascular o desconocida.

\section{Comentario}

Es muy extensa la literatura médica disponible acerca de la terapia antitrombótica, específicamente la antiagregación plaquetaria, y la prevención de eventos vasculares isquémicos, especialmente en trabajos de prevención secundaria ${ }^{1 \cdot 3}$. Está establecido que pacientes con eventos previos (IAM, ACV, AIT) se benefician con el tratamiento antiplaquetario ${ }^{3}$. Sin embargo, existían algunos puntos controvertidos referidos al balance daño/beneficio en la prevención primaria y la dosis óptima de aspirina cuando se utiliza este fármaco ${ }^{4}$. El presente trabajo documenta que los agentes antiagregantes también protegen contra eventos cardiovasculares a pacientes con riesgo aumentado por condiciones como angina crónica estable, angina inestable, claudicación intermitente, y fibrilación auricular, aún sin eventos previos.Por otro lado, refiriéndonos al rango terapéutico del AAS, especialmente en pacientes con enfermedad vasculoencefálica en que altas dosis de aspirina se relacionaron con aumento de riesgo de eventos hemorrágicos, es clarificadora la demostración de la equivalencia de eficacia entre dosis de 75 a $150 \mathrm{mg}$ y dosis mayores.Además se concluye que la antiagregación con aspirina es al menos tan efectiva como la conseguida con otros regímenes más costosos o con drogas con mucha menos historia en la práctica clínica. Es sabido que uno de los principios más elementales de nuestro accionar médico es que las intervenciones que realicemos deberán brindar beneficios exponiendo al paciente a un riesgo potencial menor que el de la patología que genera nuestra intervención. A partir de este trabajo podemos concluir que este principio queda a salvo cuando indicamos terapia antiagregante a pacientes con eventos vasculares oclusivos previos e incluso a los que sin haberlos sufrido, tienen riesgo aumentado por condiciones predisponentes. En la prevención primaria de eventos cardiovasculares en la población general (riesgo < 1\% al año), por ahora solo deberemos insistir en estimular un estilo de vida saludable interviniendo en hábitos como la dieta, la actividad física y combatiendo el tabaquismo.

Conclusión del comentador: La aspirina a una dosis de 75-150 $\mathrm{mg} /$ día es claramente beneficiosa en pacientes de riesgo cardiovascular.

\section{Dr. Alejandro Regueiro [ Clínica Médica ]}

\section{Bibliografía}

1.Dippel DW.The results of CAPRIE, IST and CAST.Thromb Res 1998;92 (Suppl 1):S13-6.

2.Meschia JF.Management of acute ischemic stroke.Posgraduate Med 2000;107 (6):85-93.

2.Meschia JF.Management of acute ischemic stroke.Posgraduate Med 2000;107 (6):85-93. 3. Antiplatelet Trialists' Collaboration. Collaborative overview of

4.Shekelle PG, MacLean CH, Morton SC, Wenger NS.Acove quality indicators. Ann Intern Med 2001;135 (8 Pt 2):653-67. 